\title{
Weighted Average Information Criterion for Selection of an Asymmetric Price Relationship
}

Doi: 10.29023/alanyaakademik.343737

\author{
Henry De-Graft ACQUAH \\ Prof. Dr., Department of Agricultural Economics and Extension, University of Cape Coast \\ (degraft.acquah@ucc.edu.gh)
}

\section{Keywords}

Price Asymmetry

Akaike's Information

Criteria (AIC)

Bayesian Information

Criteria (BIC) Weighted

Average Information

Criteria (WIC) Model

Selection

Received: 13.10 .2017

Accepted: 03.05.2018

\begin{abstract}
This study evaluates the performance of the recently developed model selection criteria (WIC) against commonly used alternatives (AIC and BIC) in terms of their ability to recover the true asymmetric data generating process. Monte Carlo simulation results indicate that the performance of the model selection methods depends on the sample size, the difference in asymmetric adjustment parameters and the amount of noise in the model used in the application. WIC outperforms AIC and BIC under stable conditions such as a large sample and small noise levels. Additionally, WIC outperforms AIC and BIC as the difference between asymmetric adjustment speeds increases. These results suggest that WIC is a very reliable and useful criterion in asymmetric price transmission model selection.
\end{abstract}

\section{INTRODUCTION}

Many competing models have been developed to measure asymmetric price transmission. Researchers modelling asymmetric price transmission are therefore faced with the decision of choosing an appropriate model among a set of competing asymmetric price transmission models. This leads to the problem of model selection. Asymmetric price transmission model selection problem involves using a model selection criteria to select the most appropriate model among computing models.

Existing criteria widely used in asymmetric price transmission model selection include Akaike Information Criteria (AIC) of Akaike (1973) and Bayesian Information Criteria (BIC) of Schwarz (1978). For example, Acquah (2010) evaluates the relative performance of AIC and BIC in discriminating among competing price transmission models. Acquah (2010) notes that AIC performed well in small samples whilst BIC, in contrast, appears to perform relatively poorly in small samples but is consistent and its performance improves with large sample size. In effect, AIC is efficient and BIC is consistent.

$\mathrm{Wu}$ and Sepulveda (1998) recently proposed a new model selection criteria, Weighted Average Information Criteria (WIC) which draws on the strengths of corrected AIC and BIC. WIC is noted to perform better than AIC and BIC in empirical applications. For example, Chen, Wu and Wang (2008) noted that WIC performs better than AIC and BIC in signal processing applications. However, little is known about the performance of WIC in asymmetric price transmission modelling context. A fundamental question which remains unanswered in asymmetric price transmission model selection is how well will the newly 
developed WIC perform when compared with commonly used AIC and BIC in asymmetric price transmission model selection. Will WIC point to the correct asymmetric price transmission model in price transmission analysis. In order to address these issues, this paper conducts a Monte Carlo study to evaluate the relative performance of the recently developed model selection criteria (WIC) against commonly used criteria (AIC and BIC) in terms of their ability to recover the true asymmetric data generating process.

The rest of the paper proceeds as follows. In the following section, an introduction of the model selection criteria is presented. This is followed by a brief description of asymmetric price transmission models. A practical application in which the performance of the model selection methods in selecting the correct asymmetric model are evaluated via Monte Carlo experimentation is presented. Finally, the study ends with conclusions.

\section{MATERIALS AND METHODS}

\subsection{Model Selection Using Information Criteria}

Information criteria is an important tool for model selection. Information theoretic model selection criteria are made up of two components. The first term is a measure of goodness of fit of the candidate model to data and the second term serves as penalty for model complexity. Fundamentally, various information criteria may differ in the way in which they penalise complexity.

\subsubsection{Akaike Information Criterion (AIC)}

Akaike (1973) proposed the Akaike Information Criteria (AIC) to estimate the expected Kullback- Leibler distance between the model generating the data and fitted candidate model. AIC can be expressed as:

$$
A I C=-2 \log (L)+2 p
$$

Where $(\mathrm{L})$ is the maximised log-likelihood of the data given the model parameter estimates and $\mathrm{p}$ is the number of parameters in the model. AIC is asymptotically efficient. However, the main problem with AIC is that it is not consistent. AIC chooses the best-approximating model to the data generating process. Models with minimum AIC values are preferred. AIC chooses the more complex model when the sample size is large.

\subsubsection{Bayesian Information Criteria (BIC)}

In order to overcome the over estimation problem of AIC, Schwarz (1978) proposed the Bayesian Information Criteria (BIC). BIC was derived using a Bayesian perspective and it selects the model with the maximum posterior probability for a given prior probability. BIC can be expressed as:

$$
B I C=-2 \log (L)+p \log (n)
$$

Where the $(\mathrm{L})$ is maximised log-likelihood of the data given the model parameter estimates, $\mathrm{p}$ is the number of parameters in the model and $n$ is the sample size. The equation of BIC differs from AIC in terms of the penalty term which is greater in BIC. BIC chooses simpler models and it is consistent. It assumes that the candidate models contain the true model and subsequently selects the true model. Notably, models with minimum BIC are preferred. 


\subsubsection{Weighted-Average Information Criterion}

$\mathrm{Wu}$ and Sepulveda (1998) recently introduced a novel model selection criteria, WeightedAverage Information Criterion (WIC) that combines the strength of corrected AIC and BIC. WIC is expressed as:

$$
W I C=-2 \log (L)+\frac{[2 n p /(n-p-1)]^{2}+(p \log n)^{2}}{2 n p /(n-p-1)+(p \log n)}
$$

Where the (L) is maximised log-likelihood of the data given the model parameter estimates, $p$ is the number of parameters in the model and $n$ is the sample size. Models with minimum WIC are preferred.

\subsection{Asymmetric Price Transmission Models}

An asymmetric price transmission model in which asymmetries specified affect the direct impact of price increases and decreases is proposed in Houck (1977) and can be represented as follows:

$$
\Delta \mathrm{y}_{t}=\beta_{1}^{+} \Delta x_{t}^{+}+\beta_{1}^{-} \Delta x_{t}^{-}+\varepsilon_{t} \quad \varepsilon_{t} \square N\left(0, \sigma^{2}\right)
$$

Where $\Delta x_{t}^{+}$and $\Delta x_{t}^{-}$are the positive and negative changes in the price series $x_{t}$ Asymmetry is tested by establishing whether the coefficients $\left(\beta_{1}^{+}\right.$and $\left.\beta_{1}^{-}\right)$are identical (that is $H_{0}: \beta_{1}^{+}=\beta_{1}^{-}$). The Houck's model assumes that the price series involved are not cointegrated.

In a context where the price series are co-integrated, Granger and Lee (1989) proposes the Error Correction Model which can be represented as follows:

$$
\Delta \mathrm{y}_{t}=\beta_{1} \Delta x_{t}+\beta_{2}^{+} E C T_{t-1}^{+}+\beta_{2}^{-} E C T_{t-1}^{-}+\varepsilon_{t}
$$

Where the price series $\mathrm{x}$ and $\mathrm{y}$ are co-integrated and the long run equilibrium relationship between them is denoted by the Error Correction Term (ECT) which is decomposed into positive and negative component. Asymmetries specified in the error correction model affect the positive and negative components of the error correction term. Symmetry in equation (5) is tested by determining whether the coefficients $\left(\beta_{2}{ }^{+}\right.$and $\left.\beta_{2}{ }^{-}\right)$are identical (that is $H_{0}: \beta_{2}{ }^{+}=\beta_{2}{ }^{-}$).

Alternatively, a complex asymmetric price transmission model in which asymmetries specified affects the direct impact of price increases and decreases, as well as positive and negative components of the error correction term, is proposed in Cramon-Taubadel and Loy (1996).

$$
\Delta \mathrm{y}_{t}=\beta_{1}^{+} \Delta x_{t}^{+}+\beta_{1}^{-} \Delta x_{t}^{-}+\beta_{2}^{+} E C T_{t-1}^{+}+\beta_{2}^{-} E C T_{t-1}^{-}+\varepsilon_{t}
$$

Where $\Delta x_{t}^{+}$and $\Delta x_{t}^{-}$are the positive and negative changes in $x_{t}$ and $\quad \boldsymbol{E C T}_{t-1}^{+}$and $E C T_{t-1}^{-}$are the positive and negative components of the error correction term. The test for 
asymmetry using the above equation is: $\boldsymbol{H}_{\mathbf{O}}: \boldsymbol{\beta}_{1}{ }^{+}=\boldsymbol{\beta}_{\mathbf{1}}{ }^{-}$and $\boldsymbol{\beta}_{2}{ }^{+}=\boldsymbol{\beta}_{2}{ }^{-}$. In this case, a joint F-test can be used to detect symmetry or asymmetry of the price transmission process.

\section{RESULTS AND DISCUSSION}

\subsection{A Comparison of the Performance of Information Criteria}

Monte simulation studies are conducted to evaluate the relative performance of a new model selection criteria (WIC) against commonly used alternative model selection criteria (AIC and BIC) in recovering the true asymmetric data generating process (DGP) under conditions of different sample sizes, levels of asymmetry and noise levels. For each Monte Carlo experiment, the data generating process is simulated from the standard error correction model as follows:

$$
\Delta y_{t}=0.7 \Delta x_{t}-0.25\left(y_{t}-x_{t}\right)^{+}{ }_{t-1}-0.75\left(y_{t}-x_{t}\right)^{-}{ }_{t-1}+\varepsilon
$$

The assignment of model parameters draws from the experimental designs of Holly et al. (2003). Subsequently, the value of $\beta_{1}$ is set to 0.7 and $\left(\beta_{2}{ }^{+}, \beta_{2}{ }^{-}\right) \in(-0.25,-0.75)$ are considered for the coefficients of the asymmetric error correction terms in the true model.

Equation 7 consists of the prices $y$ and $x$ that are generated as I (1) nonstationary variables that are co-integrated and the positive and negative deviations from the equilibrium relationship between $\mathrm{y}$ and $\mathrm{x}$.

The different asymmetric price transmission models are fitted to the simulated data and the ability of the model selection methods to recover the true model was measured and defined as the model recovery rates. The recovery rates were derived using 1000 Monte Carlo simulations. The frequency with which each model selection criteria selects the true model provides the basis for comparison. The relative performance of a new model selection criteria (WIC) and the commonly used alternative model selection criteria (AIC and BIC) are compared in terms of their success rates or ability to recover the true asymmetric data generating process (DGP) across various sample size conditions and noise levels (i.e. Model Recovery Rates). For the sake of brevity, the standard asymmetric error correction model, the complex asymmetric error correction model and the Houck's model in first differences are denoted by SECM, CECM, and HKD respectively.

The performance of the model selection methods is evaluated in terms of their ability to select the true model among a set of competing models. The results in Table 1 indicate the frequency with which each model selection criteria selects the true model.

The model selection methods studied performed reasonably well in identifying the true model, though their ability to recover the true asymmetric data generating process (DGP) increases with increase in sample size. This is consistent with previous studies (Acquah 2013; Tan \& Biswas 2012; Al-Marshadi, 2009; Markon \& Krueger, 2004; Bozdogan, 1987; Atkinson, 1980) which noted that model selection methods empirically do point to the true model. Generally, model selection performance of WIC, BIC, and AIC improved as sample sizes increased. In small samples (upper part of Table 1), the model selection methods recovered at most $81.8 \%$ of the true data generating process. When the sample size was large (lower part of Table 1), the model selection methods recovered at most $100.0 \%$ of the true model. AIC performs well in small samples, but is inconsistent and does not improve in 
performance as sample size increased whilst WIC and BIC, in contrast, are consistent and improves performance in large sample size.

Recovery rates of Weighted Average Information Criteria strongly depended on sample size for the true data generating process (DGP). It increased from 37.7 percent to 100.0 percent when the sample size was increased from 50 to 500. Similarly, recovery rates of Bayesian Information Criteria also strongly depended on sample size for the true data generating process (DGP). It increased from 81.8 percent to 99.1 percent when the sample size was increased from 50 to 500. On the other hand, recovery rates of AIC increased from 78.7 percent to 85.0 percent for the true asymmetric data generating process (DGP) when the sample size was increased from 50 to 500. Though AIC performed well in the small samples, it did not make substantial gains in recovering the true model as the sample size increased. This is consistent with the empirical findings of Seghouane and Lathauwer (2003) in signal processing modeling which indicates that AIC performs better in small samples.

The results are generally consistent with trends suggested by previous studies (Acquah 2010) which indicated that the ability of AIC to select a true model rapidly increased with sample size but at larger sample sizes it continued to exhibit a slight tendency to select complex models whilst BIC, in contrast, is consistent and improves in performance as sample size increased. Chen, Wu, and Tang (2008) note that WIC performs similarly to BIC and outperforms other criteria when the sample size is large. Generally, these results are confirmed in the Monte simulation results presented in Table 1.

Table 1. Relative performance of the model selection methods across sample size

\begin{tabular}{|c|c|c|c|c|c|}
\hline \multicolumn{2}{|c|}{ Experiment criterion } & \multicolumn{3}{|c|}{ Model fitted } & \multirow[b]{2}{*}{$\begin{array}{l}\text { SECM } \\
(\text { DGP) }(\%)\end{array}$} \\
\hline & & Methods & CECM (\%) & HKD (\%) & \\
\hline \multirow{3}{*}{$n=50$} & \multirow{3}{*}{$\sigma=1$} & AIC & 16.2 & 5.1 & 78.7 \\
\hline & & $\mathrm{BIC}$ & 5.2 & 13 & 81.8 \\
\hline & & WIC & 0 & 62.3 & 37.7 \\
\hline \multirow{3}{*}{$n=150$} & \multirow{3}{*}{$\sigma=1$} & AIC & 15.6 & 0 & 84.4 \\
\hline & & $\mathrm{BIC}$ & 3.2 & 0 & 96.8 \\
\hline & & WIC & 0 & 2.8 & 97.2 \\
\hline \multirow[b]{2}{*}{$n=500$} & \multirow[b]{2}{*}{$\sigma=1$} & AIC & 15 & 0 & 85 \\
\hline & & $\begin{array}{l}\text { BIC } \\
\text { WIC }\end{array}$ & $\begin{array}{l}0.9 \\
0\end{array}$ & $\begin{array}{l}0 \\
0\end{array}$ & $\begin{array}{l}99.1 \\
100\end{array}$ \\
\hline
\end{tabular}

Note: Recovery rates based on 1000 replications.

In order to illustrate the effects of noise level on model selection, this study considers three error sizes $(\sigma)$ ranging relatively from small to large and corresponding to 1.0, 2.0 and 3.0. Using 1000 replications, data is generated from equation (7) with the different error sizes and a sample size of 150 . The data fitting abilities of competing models are compared in relation to the true model as the error in the data generating process was increased systematically.

Table 2 shows the percentage with which each model selection criteria selects the true model (i.e. SECM) among competing models as the amount of noise in the data generating process 
was increased. Generally, the model selection performance declined with increasing amount of noise in the true asymmetric price transmission data generating process.

Recovery rates of Weighted Average Information Criteria decreased from 97.2 percent to 3.0 percent when the error size was increased from 1 to 3. Similarly, recovery rates of BIC decreased from 96.8 percent to 42.8 percent for the true data generating process (DGP) when the error size was increased from 1 to 3. Recovery rates of AIC also decreased from 85.0 percent to 65.1 percent for the true asymmetric data generating process (DGP) when the error size was increased from 1 to 3 . Except for high noise levels, WIC outperforms AIC and BIC.

Table 2. Relative performance of the selection methods across error size

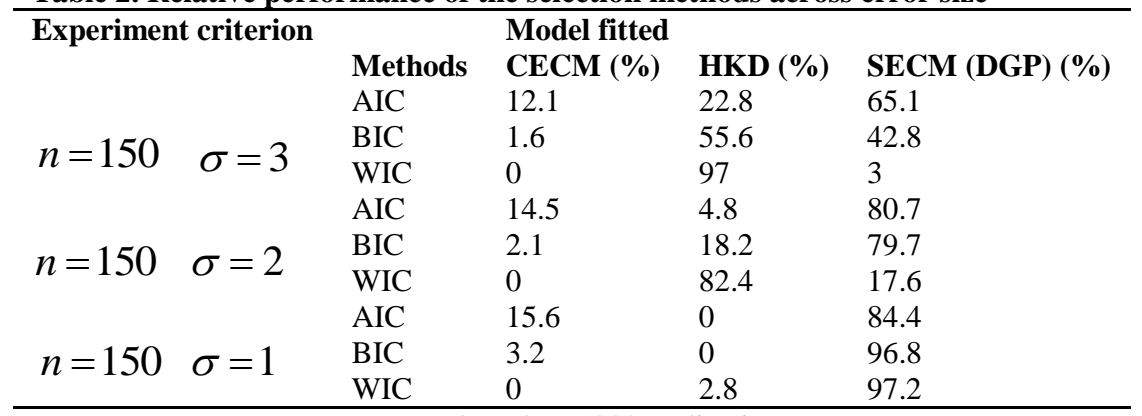

Note: Recovery rates percentages based on 1000 replications.

Simulating the effects of sample size and stochastic variance concurrently affirms that a small error and large sample improves recovery of the true asymmetric data generating process and vice versa as illustrated in Table 3 .

With a small sample of 50 and an error size of 2.0, the true data generating process was recovered at least 4.1 percent of the time by the model selection criteria as illustrated in the upper part of Table 3. On the other hand, with a relatively large sample of 150 and error size of 0.5 , at least 84.4 percent of the correct model was recovered across all the model selection methods as indicated in the lower part of Table 3. The model recovery rates of the model selection methods are derived under combined conditions of a small sample size of 50 and large error size of 2 (i.e. Unstable conditions), and a relatively large sample size of 150 and a small error size of 0.5 (i.e. Stable conditions ). Under stable conditions, model selection performance or recovery rates improved. Notably, WIC outperforms the AIC and BIC under stable conditions.

Table 3. Effects of sample size and stochastic variance on model recovery

\begin{tabular}{llllll}
\hline Experiment criterion & \multicolumn{5}{c}{ Model fitted } \\
& Methods & CECM (\%) & HKD (\%) & SECM (DGP) (\%) \\
$\sigma=2$ & \multirow{2}{*}{$*=50$} & AIC & 10.8 & 37.5 & 51.7 \\
& & BIC & 2.3 & 56.5 & 41.2 \\
& & WIC & 0 & 95.9 & 4.1 \\
& & AIC & 15.6 & 0 & 84.4 \\
\multirow{2}{*}{$n=150$} & \multirow{2}{*}{$\sigma=0.5$} & BIC & 3.2 & 0 & 96.8 \\
& & WIC & 0 & 0 & 100 \\
\hline
\end{tabular}

Note: Recovery rates based on 1000 replications.

The results are generally consistent with trends suggested by previous Monte Carlo Experimentation (Acquah, 2010) which suggest that the recovery rates of the true data generating process decrease with increasing noise levels and small sample size in asymmetric price transmission regression models. 
In order to illustrate the effects of level of asymmetry on model selection, this study simulated data of sample size 150 and error size 1 from the standard error correction model and asymmetry values of $\left(\beta_{2}{ }^{+}, \beta_{2}{ }^{-}\right) \in(-0.25,-0.50)$ or $(-0.25,-0.75)$ are considered for the coefficients of the asymmetric error correction terms. Subsequently, the effect of the increase in difference of asymmetric adjustment parameters on model recovery is examined. An increase in the difference in the asymmetric adjustment parameters from 0.25 to 0.5 culminates in an increase in model recovery of the true asymmetric data generating process as illustrated in Table 4.

Recovery rates of the Weighted Average Information Criteria respond more strongly to increases in the difference between the asymmetric adjustments parameters than other criteria (AIC and BIC).

Similarly, Cook et al. (1999) without regards to the concept of information criteria notes that the increases in the difference in asymmetric adjustments parameters from 0.25 to 0.50 have positive effects on the test for asymmetry. Importantly, the performance of the model selection methods in recovering the true data generating process depends on the difference in asymmetric adjustment parameters or speeds.

Table 4. Effects of the level of asymmetry on model recovery

\begin{tabular}{lllcl}
\hline Experiment criterion & \multicolumn{5}{c}{ Model Fitted } \\
\cline { 2 - 5 } & Methods & CECM (\%) & HKD (\%) & SECM (DGP) (\%) \\
\hline$\beta_{2}{ }^{+}-\beta_{2}{ }^{-}=0.25$ & AIC & 15.7 & 0 & 84.3 \\
& BIC & 2.9 & 1.4 & 95.7 \\
& WIC & 0 & 34.6 & 65.4 \\
$\beta_{2}{ }^{+}-\beta_{2}{ }^{-}=0.50$ & AIC & 15.6 & 0 & 84.4 \\
& BIC & 3.2 & 0 & 96.8 \\
& WIC & 0 & 2.8 & 97.2 \\
\hline
\end{tabular}

Note: Recovery rates percentages based on 1000 replications

\section{CONCLUSIONS}

This study investigated the ability of the recently developed model selection criteria (WIC) and commonly used criteria (AIC and BIC) to clearly identify the correct asymmetric price transmission model out of a set of competing models via Monte Carlo experimentation. The Monte Carlo simulations results indicated that the sample sizes, difference in asymmetric adjustment parameters and noise levels are essential in the selection of the true asymmetric price transmission model. Large sample sizes or low noise levels improve the ability of the model selection methods to identify the correct asymmetric price data generating process. Generally, WIC outperforms AIC and BIC under stable conditions such as a large sample and small noise levels. WIC performs poorly at small sample sizes but improves with increasing sample size to eventually choose the correct asymmetric price transmission model with perfect probability. Thus performing better than AIC and BIC in large samples. As the difference in asymmetric adjustment parameters increases, WIC outperforms AIC and BIC. These results suggest that WIC, which combines the strengths of AIC and BIC is a very reliable and useful criterion in asymmetric price transmission model selection. 
The comparison provided contributes to knowledge and understanding of the relative performance of recently developed WIC against commonly used AIC and BIC in an asymmetric price transmission modelling framework. Additionally, the study contributes to literature on asymmetric price transmission modelling by drawing the attention and interests of asymmetric price transmission researchers to adopt more recent statistical model selection criteria, such as WIC, in asymmetric price transmission model selection problems.

\section{REFERENCES}

ACQUAH, H. D. (2010). Comparison of Akaike information criterion and Consistent Akaike information criterion for model selection in Asymmetric Price Transmission Studies. Asian-African Journal of Economics and Econometrics. Vol. 9, No. 1, 49-56.

ACQUAH, H. D. (2013). On the Comparison of Akaike Information Criterion and Consistent Akaike Information Criterion in Selection of an Asymmetric Price Relationship: Bootstrap Simulation Results. AGRIS on-line Papers in Economics and Informatics, $5(1), 3$.

AKAIKE, H. (1973). Information Theory and an Extension of the Maximum Likelihood Principle. In: B.N. Petrov and F. Csaki (eds.) 2nd International Symposium on Information Theory. 3: 267-81 Budapest: Akademiai Kiado.

AL-MARSHADI, A. (2009) Bootstrap Simulation Procedure Applied to the Selection of Multiple Linear Regression, Journal of King Abdulaziz University: Science. Vol. 21 No 2, pp 197-212.

ATKINSON, A. C. (1980). A note on the generalized information criterion for choice of a model. Biometrika, 67, 413-418.

BOZDOGAN, H. (1987). Model Selection and Akaike's Information Criterion (AIC): The General Theory and Its Analytical Extensions. Psychometrika. 52(3):345-370.

CHEN, P., WU, T.-J., YANG, J. (2008). A comparative study of model selection criteria for the number of signals IET Radar Sonar Navig. Vol. 2, No. 3, pp. 180-188.

COOK, S., HOLLY, S., \& TURNER, P. (1999). The Power of tests for nonlinearity: the case of Granger-Lee asymmetry, Economics Letters, 62, pp.155-159.

CRAMON-TAUBADEL, V. S., \& LOY, J. P. (1996) Price Asymmetry in the International Wheat Market: Comment. Canadian Journal of Agricultural Economics, 44:311-317.

GRANGER, C. W. J., \& LEE, T. H. (1989). Investigation of Production, Sales and Inventory Relationships using Multicointegration and non-symmetric Error Correction Models. Journal of Applied Econometrics, 4:135- 159.

HOLLY, S., TURNER P., \& WEEKS, M. (2003). Asymmetric Adjustment and Bias in Estimation of an Equilibrium Relationship from a Co-integrating Regression. Computational Economics, 21:195-202.

HOUCK, J. P. (1977). An Approach to specifying and estimating nonreversible Functions. American Journal of Agricultural Economics, 59:570-572.

MARKON, K. E., \& Krueger, R. F. (2004). An Empirical Comparison of InformationTheoretic Selection Criteria for Multivariate Behavior Genetic Models. Behavior Genetics, 34, (6), pp.593- 609 
SCHWARZ, G. (1978) "Estimating the Dimension of a Model." Annals of Statistics, 6, pp. 461-464.

SEGHOUANE, A. K., \& DE LATHAUWER, L. (2003). A bootstrap model selection criterion based on Kullback's symmetric divergence. In Statistical Signal Processing, 2003 IEEE Workshop on (pp. 494-497). IEEE

TAN, M. Y. J., \& BISWAS, R. (2012). The reliability of the Akaike information criterion method in cosmological model selection. Monthly Notices of the Royal Astronomical Society, 419(4), 3292-3303.

WU, T. J., \& SEPULVEDA, A. (1998). The weighted average information criterion for order selection in time series and regression models. Statistics \& probability letters, 39(1), 1 10 . 